\title{
Beneficial Metabolic Effects of Mirabegron In Vitro and in High-Fat Diet-Induced Obese Mice
}

\author{
Lei Hao, Sheyenne Scott, Mehrnaz Abbasi, Yujiao Zu, Md Shahjalal Hossain Khan, \\ Yang Yang, Dayong Wu, Ling Zhao, and Shu Wang \\ Department of Nutritional Sciences, Texas Tech University, Lubbock, Texas (L.H., S.S., M.A., Y.Z., M.S.H.K., S.W.); Department \\ of Nutrition, University of Tennessee, Knoxville, Tennessee (Y.Y., L.Z.); and Nutrition Immunology Laboratory, Jean Mayer USDA \\ Human Nutrition Research Center on Aging, Tufts University, Boston, Massachusetts (D.W.)
}

Received December 11, 2018; accepted March 22, 2019

\begin{abstract}
Mirabegron, a $\beta 3$-adrenergic receptor agonist, has been shown to stimulate the activity of brown fat and increase the resting metabolic rate in humans. However, it is unknown whether mirabegron can reduce body weight and improve metabolic health. We investigated the antiobesity effects of mirabegron using both in vitro and in vivo models. Mouse brown preadipocytes and 3T3-L1 cells were treated with different concentrations of mirabegron $(0.03-3 \mu \mathrm{g} / \mathrm{ml})$, and the expression of brown fat-related genes was measured by quantitative real-time polymerase chain reaction. Furthermore, male C57BL/6J mice were fed a high-fat diet for 10 weeks, and mirabegron $(2 \mathrm{mg} / \mathrm{kg}$ body weight) or a vehicle control was delivered to the interscapular brown adipose tissue (BAAT) using ALZET osmotic pumps from week 7 to 10 . The metabolic parameters and tissues were analyzed. In both mouse brown preadipocytes and 3T3-L1
\end{abstract}

cells, mirabegron stimulated uncoupling protein 1 (UCP1) expression. In animal studies, mirabegron-treated mice had a lower body weight and adiposity. Lipid droplets in the iBAT of mirabegrontreated mice were fewer and smaller in size compared with those from vehicle-treated mice. H\&E staining and immunohistochemistry indicated that mirabegron increased the abundance of beige cells in inguinal white adipose tissue (iWAT). Compared with vehicletreated mice, mirabegron-treated mice had a higher gene expression of UCP1 (14-fold) and cell death-inducing DNA fragmentation factor alpha-like effector A (CIDEA) (4-fold) in iWAT. Furthermore, mirabegron-treated mice had improved glucose tolerance and insulin sensitivity. Taken together, mirabegron enhances UCP1 expression and promotes browning of iWAT, which are accompanied by improved glucose tolerance and insulin sensitivity and prevention from high-fat diet-induced obesity.

\section{Introduction}

Obesity affects $35.0 \%$ of adult men and $40.4 \%$ of adult women in the United States (Flegal et al., 2016). Obesity increases the risk of several chronic diseases such as type 2 diabetes, heart disease and even some types of cancers. In addition, obesity creates a huge health care costs burden. A metaanalysis shows that the United States spent $\$ 149.4$ billion annually on obesity-related health care between 2008 and 2012 (Kim and Basu, 2016), and the number is expected to rise over the next few decades (Cawley and Meyerhoefer, 2012).

Two kinds of adipose tissue exist in mammals, with distinct physiologic functions: white adipose tissue (WAT), which is specialized for the storage of excessive triglycerides, and brown adipose tissue (BAT), which plays a central role in

This work was supported by the College of Human Sciences of Texas Tech University and the National Institutes of Health National Center for Complementary \& Integrative Health (Grant R15AT008733).

https://doi.org/10.1124/jpet.118.255778. metabolizing glucose, fatty acids, and other chemicals to produce heat (Cannon and Nedergaard, 2004; Ozaki et al., 2011; Kazak et al., 2015). The brown-like adipocytes found in WAT are called brite/beige adipocytes. Similar to classic brown adipocytes in BAT, beige adipocytes possess multilocular lipid droplets, a large number of mitochondria, and unique brown fat gene expression markers such as uncoupling protein 1 (UCP1), cell death-inducing DNA fragmentation factor alpha-like effector A (CIDEA), and peroxisome proliferator-activated receptor $\gamma(\operatorname{PPAR} \gamma)$ coactivator $1 \alpha$ $(\mathrm{PGC} 1 \alpha)$ (Harms and Seale, 2013).

Traditionally, it was thought that BAT only existed in rodents and human infants. In 2009, several studies using positron emission tomography scanning showed that human adults do have active BAT (Cypess et al., 2009; van Marken Lichtenbelt et al., 2009; Virtanen et al., 2009). The fact that activation of BAT can increase energy expenditure makes BAT a promising new target for obesity and type 2 diabetes treatment.

ABBREVIATIONS: $\beta 3$-AR, $\beta 3$ adrenergic receptor; BAT, brown adipose tissue; BRL37344, sodium;2-[4-[(2R)-2-[[(2R)-2-(3-chlorophenyl)-2hydroxyethyl]amino]propyl]phenoxy]acetate; CHD, chow diet; CIDEA, cell death-inducing DNA fragmentation factor alpha-like effector A; CL316243, disodium; 5-[(2R)-2-[[(2R)-2-(3-chlorophenyl)-2-hydroxyethyl]amino]propyl]-1, 3-benzodioxole-2,2-dicarboxylate; CPT1, carnitine palmitoyltransferase 1; DEX, dexamethasone; HFD, high-fat diet; HSL, hormone-sensitive lipase; iBAT interscapular BAT; IBMX, 3-isobutyl-1methylxanthine; iWAT, inguinal white adipose tissue; MA, mirabegron; PCR, polymerase chain reaction; PGC-1 $\alpha$, PPAR- $\gamma$ coactivator $1 \alpha$; PPAR $\gamma$, peroxisome proliferator-activated receptor $\gamma$; PRDM16, PR domain containing 16; P/S, penicillin/streptomycin; TMEM26, transmembrane protein 26; UCP1, uncoupling protein 1; VEGFa, vascular endothelial growth factor a; VEH, vehicle; WAT, white adipose tissue. 
Most medications approved by U.S. Food and Drug Administration for the treatment of obesity reduce energy intake by either suppressing appetite or reducing fat absorption in the intestine (Arch, 2015; Omran, 2017). However, none of the antiobesity drugs targets energy expenditure through stimulating BAT activity. Several strategies, such as genetic manipulations, pharmaceutic agents, dietary factors, and cold challenge, have been used to promote BAT activity or the browning of WAT (Merlin et al., 2016). One area of focus has been the promotion of energy expenditure through activation of $\beta 3$-adrenergic receptor ( $\beta 3$-AR) on brown adipocytes (Arch, 2011).

Human $\beta 3$-AR was identified in 1989 (Emorine et al., 1989), and it was shown to be expressed in many tissues, including WAT, BAT, urinary bladder, heart, and other tissues (Ursino et al., 2009). The adrenergic signaling regulates the activity of enzymes involved in lipolysis and fuel utilization in BAT (Townsend and Tseng, 2014). A number of animal studies have shown that $\beta 3$-AR agonists have antiobesity and/or antidiabetic effects (Umekawa et al., 1997; Kiso et al., 1999; Williams et al., 1999; Arch, 2002). However, most of these $\beta 3$-AR agonists such as disodium;5-[(2R)-2[[(2R)-2-(3-chlorophenyl)-2-hydroxyethyl]amino]propyl]-1,3benzodioxole-2,2-dicarboxylate (CL316243), ritobegron, solabegron, and sodium;2-[4-[(2R)-2-[((2R)-2-(3-chlorophenyl)2-hydroxyethyl]amino]propyl]phenoxy]acetate (BRL37344), have not been approved for the treatment of obesity and its related metabolic disorders due to their low efficacy and high toxicity in humans (Arch, 2011).

Mirabegron is a $\beta 3$-AR agonist that was approved for treating overactive bladder in the United States in 2012 (Sacco et al., 2014). Several randomized, double-blind, and placebocontrolled studies have shown that the adverse events were comparable between mirabegron-treated and placebo groups (Yamaguchi et al., 2015; Herschorn et al., 2017; Tubaro et al., 2017). Mirabegron was shown to be well tolerated and to have higher patient compliance due to its fewer side effects compared with the anticholinergic drugs (Dehvari et al., 2018). More interestingly, one recent study showed that mirabegron $(200 \mathrm{mg})$ acutely stimulated the activity of BAT thermogenesis and raised the resting metabolic rate in healthy individuals (Cypess et al., 2015). However, this dose is much higher than the recommended daily dose $(50 \mathrm{mg})$ for treating overactive bladder (Cypess et al., 2015). An animal study demonstrated that acute mirabegron administration $(0.1 \mu \mathrm{g} / \mathrm{g}$ body weight) through intraperitoneal injection to mice stimulated glucose uptake in BAT (Roberts-Toler et al., 2015).

All these studies suggest that mirabegron may have beneficial metabolic effects in humans. However, it is still unknown whether mirabegron can stimulate brown adipocyte formation or targeted administration of mirabegron to BAT can reduce body weight and improve metabolic functions such as glucose tolerance and insulin sensitivity. To address these issues, in this study we investigated the effects of mirabegron on obesity and glucose homeostasis in vitro using mouse brown preadipocyte cells and 3T3-L1 white preadipocytes and in vivo using C57BL/6J mice fed a high-fat diet.

\section{Materials and Methods}

Culturing of Murine Brown Preadipocytes and Treatments. The murine brown preadipocyte cell line was provided by
Dr. Johannes Klein, who developed the cell line from the fibrostromal fraction of iBAT of newborn C57BL/6 mice (Klein et al., 2002). The method to culture brown preadipocytes was previously described elsewhere (Hao et al., 2018). Briefly, mouse brown preadipocytes were maintained in regular growth medium containing DMEM with $20 \% \mathrm{FBS}$ at $37^{\circ} \mathrm{C}$ in a humidified incubator with $5 \% \mathrm{CO}_{2}$, until they reached confluence. To induce differentiation, the cells were initially incubated in induction medium containing DMEM supplemented with $20 \% \mathrm{FBS}, 20 \mathrm{nM}$ insulin, $1 \mathrm{nM}$ triiodothyronine, $0.125 \mathrm{mM}$ indomethacin, $2 \mu \mathrm{g} / \mathrm{ml}$ dexamethasone (DEX), and $0.5 \mathrm{mM}$ 3-isobutyl-1-methylxanthine (IBMX). After 48 hours, the cells were incubated in differentiation medium containing DMEM supplemented with $20 \%$ FBS, $20 \mathrm{nM}$ insulin, and $1 \mathrm{nM}$ triiodothyronine for 6 days. The differentiation medium was changed every 48 hours.

This cell line expresses $\beta 3$-AR after differentiation (our pilot study). To study the effects of mirabegron on brown preadipocytes, mirabegron $(0.03,0.3$, and $3 \mu \mathrm{g} / \mathrm{ml})$ (Santa Cruz Biotechnology, Inc., Dallas, TX), or the vehicle control dimethyl sulfoxide (DMSO), or the positive control CL316243 $(0.3 \mu \mathrm{g} / \mathrm{ml})$, a selective $\beta 3$-AR agonist (Klein et al., 1999), was added to the differentiation medium and replaced with each change of the differentiation medium during the differentiation process (6 days).

TABLE 1

Primer sequences for both mouse brown and white preadipocytes and C57BL/6J mice

\begin{tabular}{|c|c|c|}
\hline \multirow{3}{*}{$\begin{array}{l}\text { Gene Name } \\
36 \mathrm{~B} 4\end{array}$} & \multicolumn{2}{|r|}{ Primer Sequences } \\
\hline & Forward & GCTTCGTGTTCACCAAGGAGGA \\
\hline & Reverse & GTCCTAGACCAGTGTTCTGAGC \\
\hline \multirow[t]{2}{*}{ UCP1 } & Forward & GCTTTGCCTCACTCAGGATTGG \\
\hline & Reverse & CCAATGAACACTGCCACACCTC \\
\hline \multirow[t]{2}{*}{$\mathrm{DIO} 2$} & Forward & CCAGCGGAGGCAGAAGATAG \\
\hline & Reverse & CCAGTTTAACCTGTTTGTAGGCA \\
\hline \multirow[t]{2}{*}{$\operatorname{PPAR} \gamma$} & Forward & GTACTGTCGGTTTCAGAAGTGCC \\
\hline & Reverse & ATCTCCGCCAACAGCTTCTCCT \\
\hline \multirow[t]{2}{*}{$\mathrm{PGC} 1 \alpha$} & Forward & GAATCAAGCCACTACAGACACCG \\
\hline & Reverse & CATCCCTCTTGAGCCTTTCGTG \\
\hline \multirow[t]{2}{*}{ PRDM16 } & Forward & ATCCACAGCACGGTGAAGCCAT \\
\hline & Reverse & ACATCTGCCCACAGTCCTTGCA \\
\hline \multirow{2}{*}{ CIDEA } & Forward & AGAAGGTCCTACTGACCCCC \\
\hline & Reverse & ACCCGGTGTCCATTTCTGTC \\
\hline \multirow[t]{2}{*}{ LPL } & Forward & TCCAGAGTTTGACCGCCTTC \\
\hline & Reverse & CCAGCTGGATCCAAACCAGT \\
\hline \multirow[t]{2}{*}{ NRF-1 } & Forward & GGCAACAGTAGCCACATTGGCT \\
\hline & Reverse & GTCTGGATGGTCATTTCACCGC \\
\hline \multirow[t]{2}{*}{ ATGL } & Forward & TTGTCCTGCCCCACTAAGAG \\
\hline & Reverse & ACTCAGAGAACCCCGTGAAG \\
\hline \multirow[t]{2}{*}{ HSL } & Forward & AGACACTTCGCTGTTCCTCA \\
\hline & Reverse & CTTCAGCCTCTTCCTGGGAA \\
\hline \multirow[t]{2}{*}{ CPT1 } & Forward & GGACTCCGCTCGCTCATT \\
\hline & Reverse & GAGATCGATGCCATCAGGGG \\
\hline \multirow[t]{2}{*}{ MCAD } & Forward & TCAAGATCGCAATGGGTGCT \\
\hline & Reverse & GCTCCACTAGCAGCTTTCCA \\
\hline \multirow[t]{2}{*}{ COXII } & Forward & GCTCTCCCCTCTCTACGCAT \\
\hline & Reverse & AGCAGTCGTAGTTCACCAGG \\
\hline \multirow[t]{2}{*}{$18 \mathrm{~S}$} & Forward & GATTCTCCGTTACCCGTCAA \\
\hline & Reverse & CGTGCTGACGACAGATCACT \\
\hline \multirow[t]{2}{*}{ ELOVL3 } & Forward & ACAGAGGCACACACAAACAC \\
\hline & Reverse & GATAGGGAAGCAGGGTCTCC \\
\hline \multirow[t]{2}{*}{ Leptin } & Forward & TGGGGTTTTGGAGCAGTTTG \\
\hline & Reverse & CTGTCACTCTTTCCCGGTCT \\
\hline \multirow[t]{2}{*}{ CD137 } & Forward & CGTGCAGAACTCCTGTGATAAC \\
\hline & Reverse & GTCCACCTATGCTGGAGAAGG \\
\hline \multirow[t]{2}{*}{ TMEM26 } & Forward & ACCCTGTCATCCCACAGAG \\
\hline & Reverse & TGTTTGGTGGAGTCCTAAGGTC \\
\hline
\end{tabular}

ATGL, adipose triglyceride lipase; COXII, cytochrome c oxidase subunit 2; CPT1, carnitine palmitoyltransferase 1; DIO2, type II iodothyronine deiodinase; DIO2, type II iodothyronine deiodinase; ELOVL3, elongation of very long chain fatty acids protein 3; HSL, hormone-sensitive lipase; LPL, lipoprotein lipase; MCAD, medium-chain acyl-CoA dehydrogenase; NRF-1, nuclear respiratory factor 1 ; PGC- $1 \alpha$, PPAR- $\gamma$ coactivator $1 \alpha$; PPAR $\gamma$, peroxisome proliferator-activated receptor $\gamma$; PRDM16, PR domain containing 16; TMEM26, transmembrane protein 26 ; UCP1, uncoupling protein 1. 
Culturing of Mouse 3T3-L1 Adipocytes and Treatments. Mouse 3T3-L1 preadipocytes purchased from the American Type Culture Collection (ATCC, Manassas, VA) were grown in DMEM supplemented with $10 \% \mathrm{FBS}$ and $1 \%$ penicillin/streptomycin (P/S). To induce differentiation, the cells were cultured in DMEM containing $10 \% \mathrm{FBS}, 1 \% \mathrm{P} / \mathrm{S}, \operatorname{IBMX}(0.5 \mathrm{mM})$, DEX $(1 \mu \mathrm{M})$, and insulin $(10 \mu \mathrm{g} / \mathrm{ml})$ for 3 days. The cells then were incubated in DMEM with $10 \%$ FBS, $1 \% \mathrm{P} / \mathrm{S}$, and insulin $(10 \mu \mathrm{g} / \mathrm{ml})$ for 2 days. Subsequently, the cells were maintained in DMEM supplemented with $10 \% \mathrm{FBS}$ and $1 \% \mathrm{P} / \mathrm{S}$ for an additional 2 days.

During the last day of differentiation, cells were treated with DMSO (vehicle control), mirabegron ( $3 \mu \mathrm{g} / \mathrm{ml}$ ), or the positive control isoproterenol (ISO) $(1 \mu \mathrm{M})$, a $\beta 3$-AR agonist (Asano et al., 2014), for 6 hours before the cells were harvested for total RNA isolation. In addition, a subset of cells were only maintained in basal medium containing DMEM with $10 \% \mathrm{FBS}$ and $1 \% \mathrm{P} / \mathrm{S}$ as a negative differentiation control.

Animals. Male C57BL/6J mice (6 weeks old) were purchased from the Jackson Laboratory (Bar Harbor, ME) and housed in rooms with the temperature set at $22^{\circ} \mathrm{C}$ and a 12-hour light/dark cycle. The mice were initially group-housed (five/cage) and had ad libitum access to food and water. After 1 week of acclimation, the mice were fed a high-fat diet (HFD) (D12492; Research Diets, New Brunswick, NJ) for 6 weeks.

The mice (13 weeks old) were randomized and divided into the following two groups (six mice per group): a mirabegron (MA) group (HFD + MA) and a vehicle control group that received DMSO (50\%, vol\%) and polyethylene glycol $(50 \%$, vol $\%)(\mathrm{HFD}+\mathrm{VEH})$. Mirabegron $(10 \mathrm{mg} / \mathrm{ml})$ dissolved in DMSO (50\%, vol\%) and polyethylene glycol (50\%, vol\%) was put into ALZET osmotic pumps (model 2004; Durect Corporation, Cupertino, CA), which infused $0.25 \mu \mathrm{l} / \mathrm{h}$ of mirabegron, approximating $2 \mathrm{mg} / \mathrm{kg}$ per day. The dosage of mirabegron was based on our pilot study and the literature on CL316243 in rodent studies (Xiao et al., 2015; Warner et al., 2016). The pumps were implanted subcutaneously on the back as previously described elsewhere (Hao et al., 2018). The mice were individually housed after the implantation.

Control C57BL/6J mice were fed chow diet (CHD) (2020X; Envigo, Indianapolis, IN) during the whole experiment. The food intake and body weight of all the mice were measured every week. Four weeks after implantation, the mice were fasted for 6 hours then euthanized by $\mathrm{CO}_{2}$ asphyxiation. Blood was drawn from the liver portal vein and kept in EDTA-coated tubes. Plasma was separated from the blood by centrifugation at $2000 \mathrm{~g}$ for 20 minutes at $4^{\circ} \mathrm{C}$. The organs and tissues were rapidly dissected, weighed, flash-frozen in liquid nitrogen, and stored at $-80^{\circ} \mathrm{C}$ until analysis. A portion of iBAT and WAT was fixed with $10 \%$ phosphate-buffered formalin for histologic and immunohistochemistry analysis. The Institutional Animal Care \& Use Committee of Texas Tech University approved all animal procedures (protocol number: 16028).

Plasma Lipid Profile. Plasma concentrations of high-density lipoprotein-cholesterol, low-density lipoprotein-cholesterol, very-lowdensity lipoprotein-cholesterol, triglycerides, and total cholesterol were measured as previously described elsewhere (Hao et al., 2018).

H\&E Staining. Portions of iBAT and inguinal white adipose tissue (iWAT) were fixed with $10 \%$ phosphate-buffered formalin and embedded in paraffin. The H\&E staining was performed by the Department of Pathology of the Texas Tech University Health Sciences Center as previously described elsewhere (Hao et al., 2018). The images were taken under an Evos microscope (AMG, Bothell, WA). The neutral lipid occupied surface area of iBAT was quantified with ImageJ software (U.S. National Institutes of Health, Bethesda, MD).
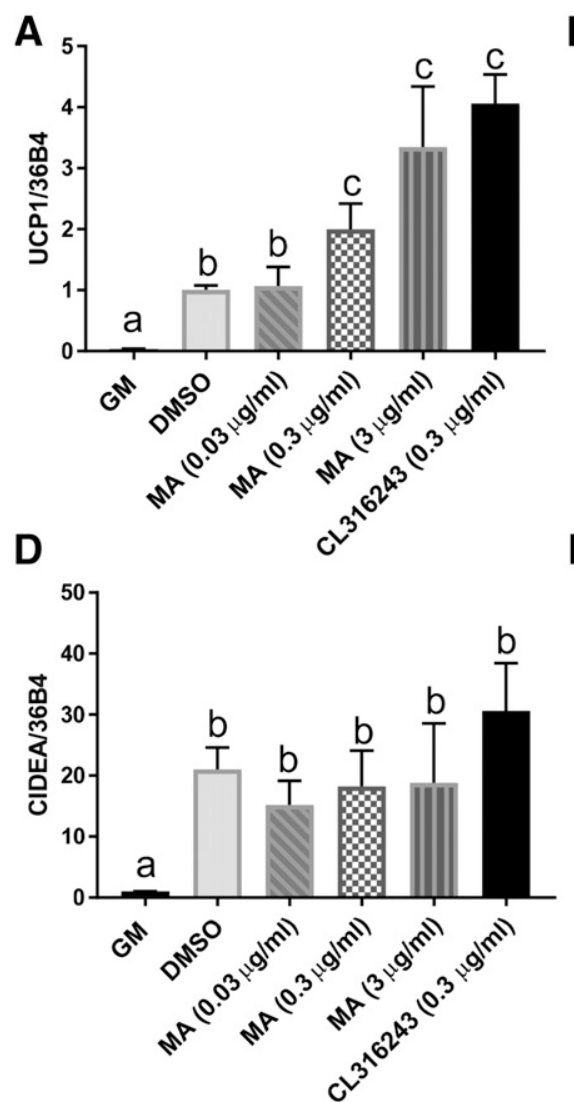
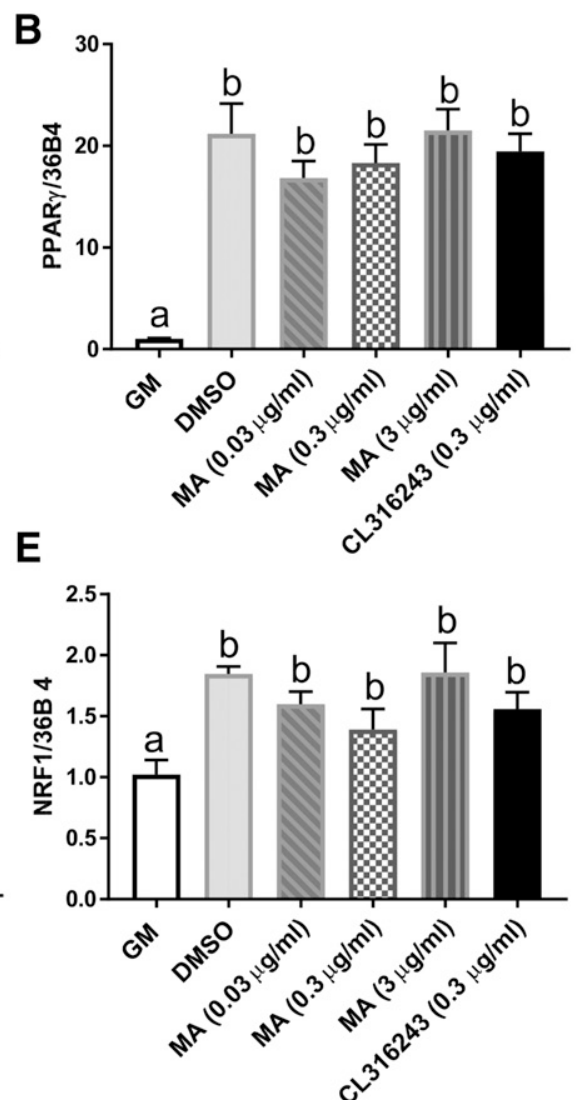

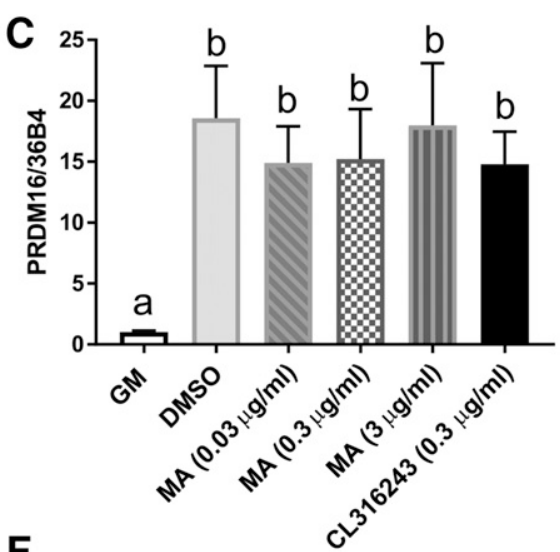

$\mathbf{F}$

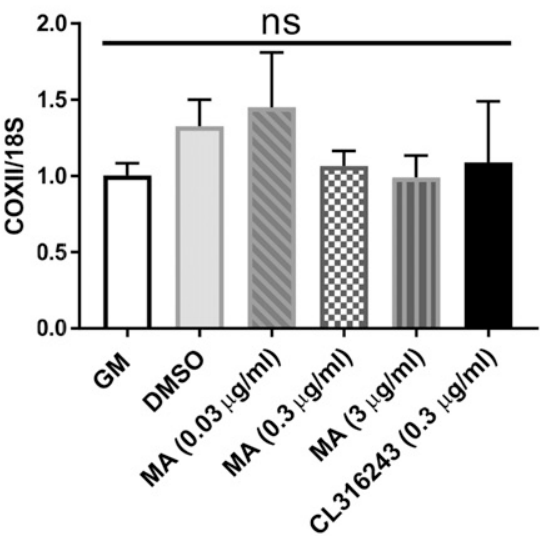

Fig. 1. Mirabegron-induced UCP1 gene expression in mouse brown preadipocytes. Gene expression of brown fat cell differentiation markers: (A) UCP1, (B) PPAR $\gamma$, (C) PRDM16, (D) CIDEA, (E) nuclear respiratory factor 1 (NRF1), and (F) DNA copies of cytochrome c oxidase subunit 2 (COXII). DMSOtreated cells were set as fold 1. All genes were normalized with 36B4. Data are presented as mean \pm S.E.M. $(n=3)$. Bars without a common superscript differ, $P<0.05$, and ns is not statistically significant. 
Immunohistochemistry. The formalin-fixed tissue was embedded in paraffin and cut to $5-\mu \mathrm{m}$ sections by the Department of Pathology of the Texas Tech University Health Sciences Center. Deparaffinized and rehydrated sections were incubated with antiUCP1 antibody produced in rabbit (U6382, 1000× dilution; SigmaAldrich, St. Louis, MO) overnight at $4^{\circ} \mathrm{C}$, followed by incubation with biotinylated secondary antibody for 1 hour. Then the sections were developed using avidin-conjugated horseradish peroxidase with diaminiobenzidine as the substrate by using a Vectastain ABC kit (Vector Laboratories, Burlingame, CA) in accordance with the manufacturer's instructions. After development, the slides were mounted under coverslips with Permount, and images were taken under an Evos microscope (AMG) after 24 hours of drying. The UCP1 intensity was quantified via a reciprocal intensity method described previously elsewhere (Nguyen et al., 2013).

Quantitative Real-Time Polymerase Chain Reaction. Total RNA was extracted using TRIzol reagent (ThermoFisher Scientific, Waltham, MA) according to the manufacturer's instructions. The total RNA abundance was measured using a NanoDrop ND-1000 spectrophotometer (NanoDrop Technologies, Wilmington, DE). Complementary DNA was synthesized by using a Maximal First Strand cDNA Synthesis Kit for real-time quantitative polymerase chain reaction (PCR) with dsDNase (\#K1671; Thermo Scientific, Pittsburgh, $\mathrm{PA})$ according to the manufacturer's instructions.

Quantitative real-time PCR was performed by using PowerUp SYBR Green Master Mix (Applied Biosystems, Austin, TX) in Hard-Shell 96-well skirted PCR plates (Bio-Rad Laboratories, Hercules, CA) and an Eppendorf Mastercycler ep realplex instrument. The PCR cycle conditions were $50^{\circ} \mathrm{C}$ for 2 minutes, $95^{\circ} \mathrm{C}$ for 2 minutes, then 40 cycles of $95^{\circ} \mathrm{C}$ for 15 seconds and $60^{\circ} \mathrm{C}$ for 1 minute. The relative gene expression was calculated by the comparative $2^{-\Delta \Delta \mathrm{Ct}}$ method using $36 \mathrm{~B} 4$ as a reference gene. The sequences of forward and reverse primers are listed in Table 1.

Body Composition. The body composition analysis was performed at weeks 7 and 10 by using an EchoMRI Body Composition
Analyzer (EchoMRI LLC, Houston, TX) according to the manufacturer's instructions.

Glucose and Insulin Tolerance Tests. After 2 weeks of treatments, an intraperitoneal glucose tolerance test (IPGTT) was performed on awake mice fasted for 6 hours by intraperitoneal injection of glucose ( $2 \mathrm{~g} / \mathrm{kg}$ body weight). After 3 weeks of treatments, an insulin tolerance test (ITT) was performed on awake mice fasted for 4 hours by intraperitoneal injection of Humulin-R-insulin $(0.75 \mathrm{U} / \mathrm{kg}$ body weight) (Lilly USA, Indianapolis, IN). Blood glucose concentrations were measured from the tail vein by using a OneTouch Ultra2 glucometer (LifeScan Europe, Zug, Switzerland) at baseline and at $15,30,60,90$, and 120 minutes after injection.

Statistical Analysis. All data were analyzed by using Prism 7 (GraphPad Software, San Diego, CA) and are presented as mean \pm S.E.M. One-way ANOVA with repeated measures followed by Newman-Keuls multiple comparisons test was performed to determine the differences among groups. $P<0.05$ was considered statistically significant.

\section{Results}

Mirabegron Enhanced UCP1 Expression in Mouse Brown Preadipocytes. C57BL/6J mouse brown preadipocytes were treated with different concentrations of mirabegron $(0.03,0.3$, and $3 \mu \mathrm{g} / \mathrm{ml})$ during cell differentiation. Mirabegron enhanced UCP1 expression in a dose-dependent manner. As compared with the vehicle control (DMSO), mirabegron at the levels of 0.3 and $3 \mu \mathrm{g} / \mathrm{ml}$ significantly increased UCP1 expression (Fig. 1A). CL316243, a $\beta 3$-agonist as a positive control, also increased UCP1 gene expression.

Neither mirabegron nor CL316243 significantly changed the expression of other brown adipocyte-related markers, such
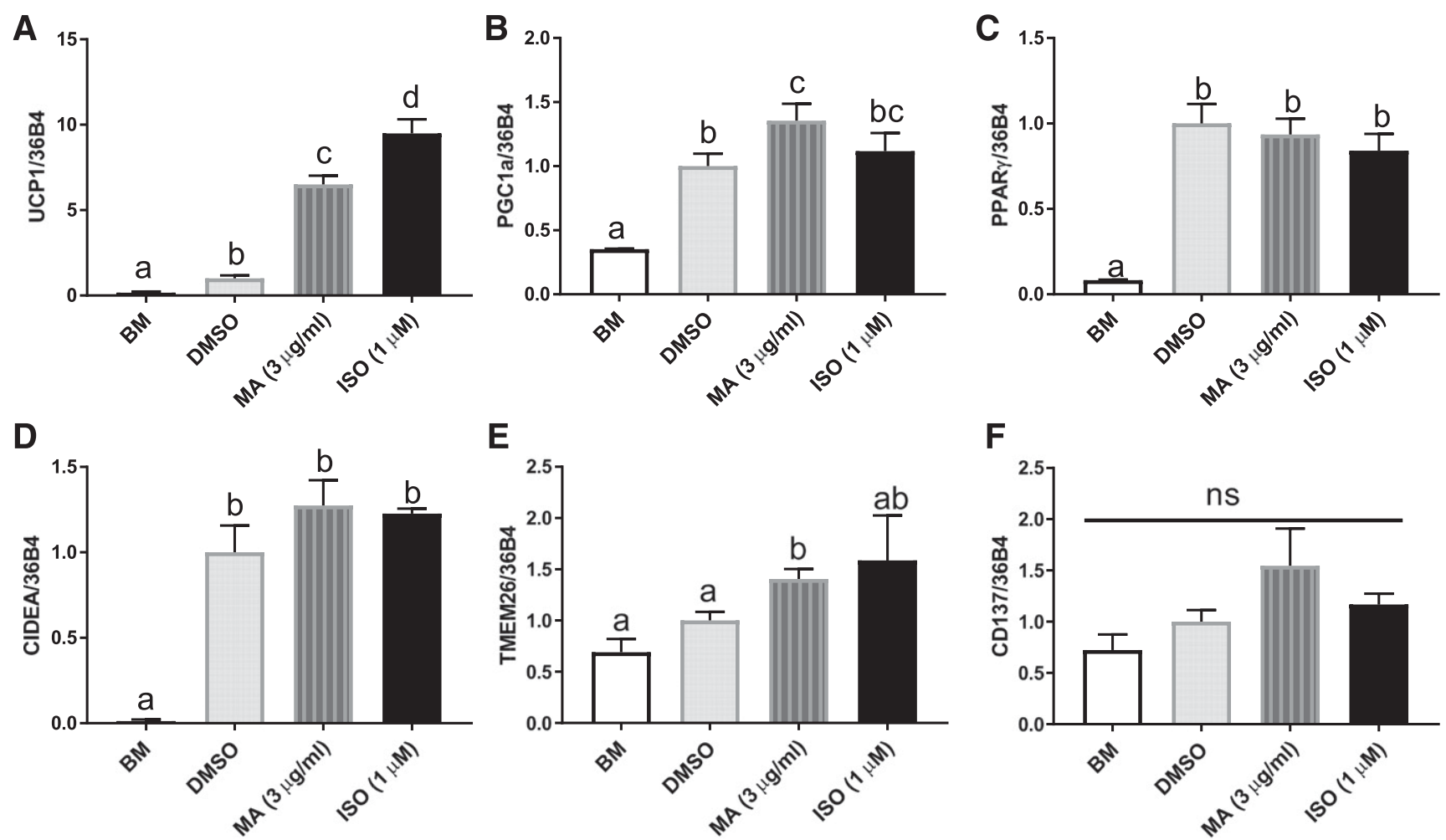

Fig. 2. Mirabegron increased the gene expression of browning-related markers in 3T3-L1 adipocytes. Gene expression of browning related markers: (A) UCP1, (B) PGC1 $\alpha$, (C) PPAR $\gamma$, (D) CIDEA, (E) TMEM26, and (F) CD137. DMSO-treated cells were set as fold 1. All genes were normalized with 36B4. Data are presented as mean \pm S.E.M. $(n=3)$. Bars without a common superscript differ, $P<0.05$, and ns is not statistically significant. 
as PPAR $\gamma$, PR domain containing 16 (PRDM16), CIDEA, and nuclear respiratory factor 1 (NRF-1) (Fig. 1, B-E), or cytochrome c oxidase subunit 2 (COXII), a mitochondrial biogenesis marker (Fig. 1F). The mRNA levels of all tested brown adipocyte differentiation markers were significantly increased in cells treated with the vehicle control (DMSO) compared with the cells only exposed to growth medium, which demonstrated the successfulness of cell differentiation (Fig. 1, A-E).

Mirabegron Increased Gene Expression of BrowningRelated Markers in 3T3-L1 Adipocytes. We further investigated the browning effect of mirabegron in differentiated 3T3-L1 white adipocytes in vitro. Mature 3T3-L1 adipocytes were treated with the vehicle DMSO, mirabegron $(3 \mu \mathrm{g} / \mathrm{ml})$, or isoproterenol $(1 \mu \mathrm{M})$, a $\beta 3$-agonist as a positive control for 6 hours. UCP1 gene expression was significantly elevated by 5.5-fold with mirabegron treatment compared with the vehicle control (DMSO) (Fig. 2A). PGC1 $\alpha$, one of the key regulators of browning, was also significantly up-regulated by mirabegron treatment compared with the vehicle control (DMSO) (Fig. 2B).

There were no differences found in PPAR $\gamma$ and CIDEA expression between mirabegron and control (Fig. 2, C and D). Expression of transmembrane protein 26 (TMEM26) and CD137, two common markers for beige adipocytes, were also measured. TMEM26 expression was increased with mirabegron treatment compared with the vehicle control (Fig. 2E), but there were no differences in CD137 expression (Fig. 2F).

Mirabegron Reduced Body Weight Gain in Mice Fed HFD. Mice in the HFD + VEH group had significantly higher body weight and fat mass compared with those in the CHD group (Fig. 3, A and B). Mice in the HFD + MA group had
$12 \%$ lower body weight and $25 \%$ lower fat mass than those in the HFD + VEH group (Fig. 3, A-C), although their food intake was not different. Compared with the mice treated with HFD + VEH, the mice treated with HFD + MA had significantly lower weight of iWAT, gonadal WAT (gWAT), and retroperitoneal WAT (rWAT) (Fig. 3D), while no significant difference was found in the iBAT mass (Fig. 3D). In addition, significantly improved glucose tolerance (Fig. 3E) and insulin sensitivity (Fig. 3F) were observed after 2 and 3 weeks of mirabegron treatment, respectively. Mirabegron had no effect on the plasma levels of cholesterol and triglyceride (data not shown).

Mirabegron Reduced iBAT Whitening in Mice Fed HFD. As compared with the CHD group, iBAT in the HFD + VEH group became filled with large lipid droplets, a phenotype called "whitening" and the lipid occupied surface area was significantly decreased in the HFD+MA group relative to the HFD + VEH group (Fig. 4, A and B). There were no differences in the mRNA expression of UCP1, PGC1 $\alpha, \operatorname{PPAR} \gamma$, and lipoprotein lipase (Fig. 4, C-F) in iBAT between HFD + VEH and HFD + MA groups. HFD + MA compared with $\mathrm{HFD}+\mathrm{VEH}$ had an insignificant 2-fold increase in type II iodothyronine deiodinase (DIO2) gene expression in iBAT (Fig. 4G).

Mirabegron Induced iWAT Browning in Mice Fed HFD. H\&E staining revealed that HFD + MA noticeably increased the abundance of multilocular adipocytes in iWAT, which is a hallmark of WAT browning (Fig. 5A). Immunohistochemistry showed that UCP1 protein in iWAT of mice treated with HFD + MA was more abundant compared with that in the HFD + VEH group (Fig. 5, B and C). Consistent
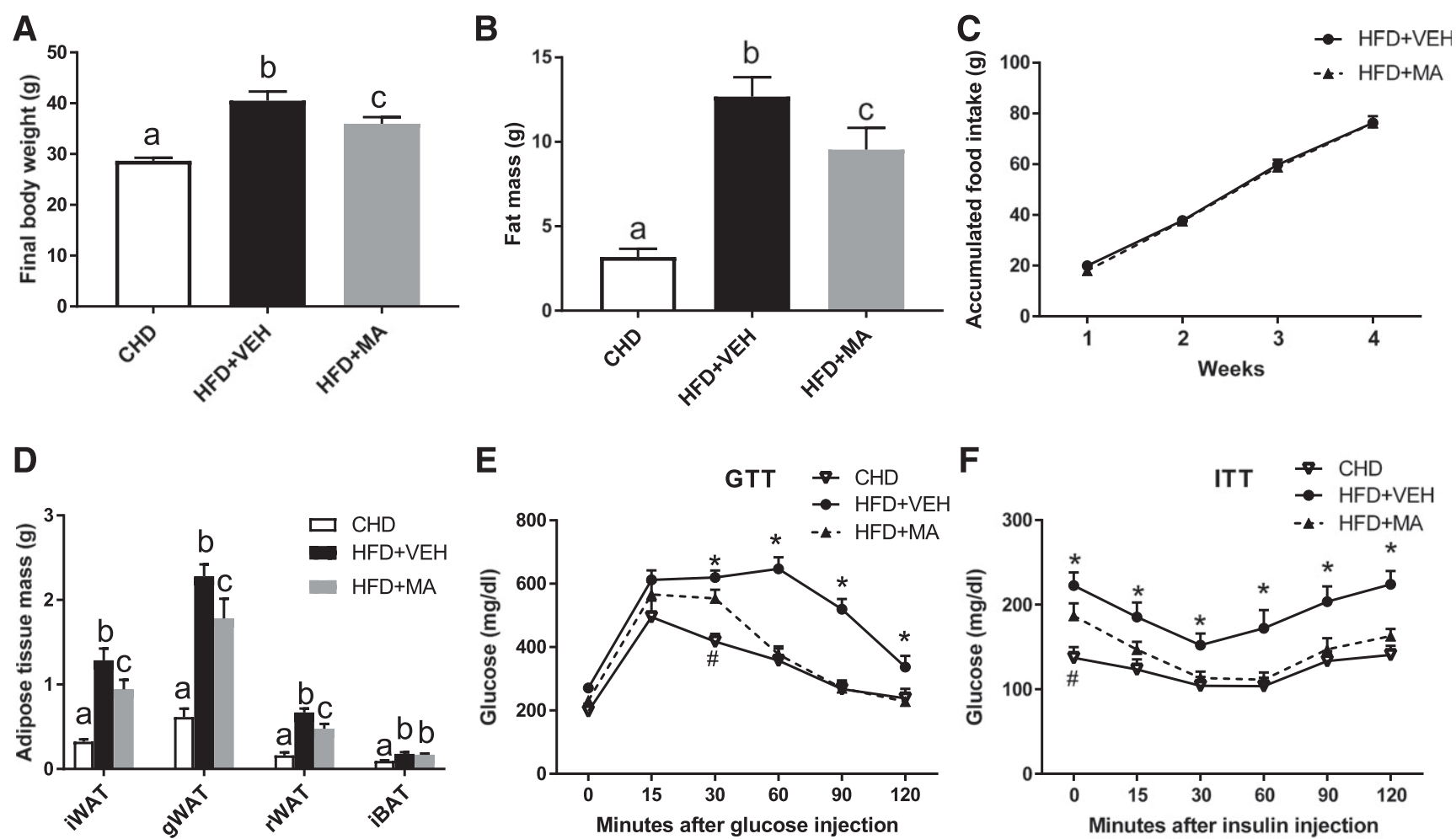

Fig. 3. Mirabegron reduced body weight gain in mice fed a HFD. (A) Final body weight. (B) Fat mass. (C) Accumulated food intake per mouse. (D) Adipose tissue weight in different fat depots. (E) Glucose tolerance test (GTT). (F) Insulin tolerance test (ITT). Data are presented as mean \pm S.E.M. $(n=6) .{ }^{*} P<0.05$ compared with the HFD + VEH group. ${ }^{\sharp} P<0.05$ compared with the HFD + MA group. Bars without a common superscript differ, $P<0.05$. 
A

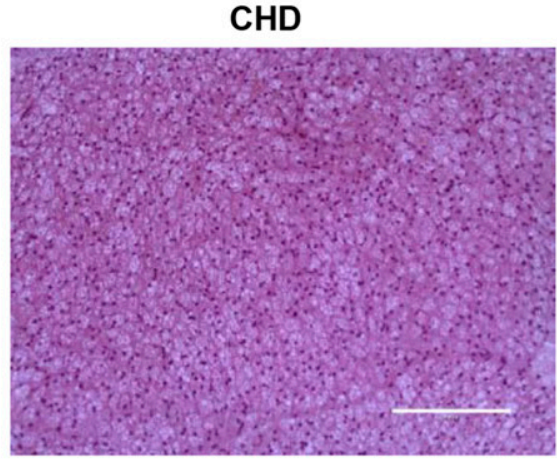

B

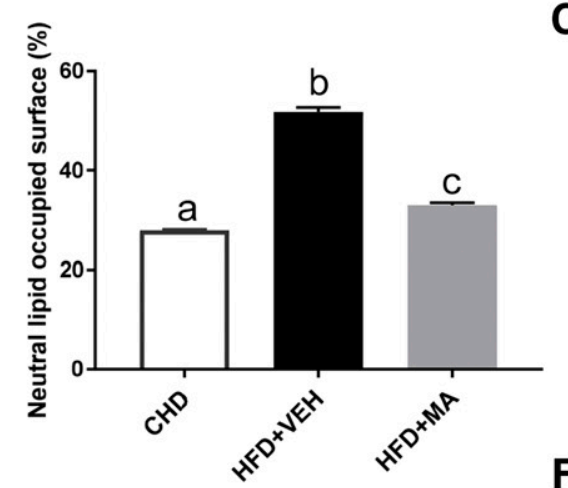

E

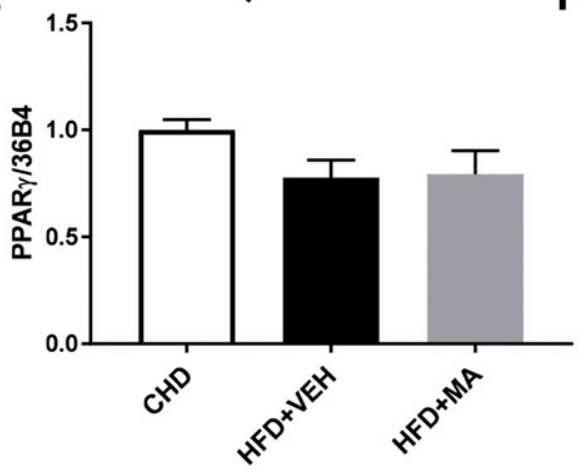

HFD+VEH

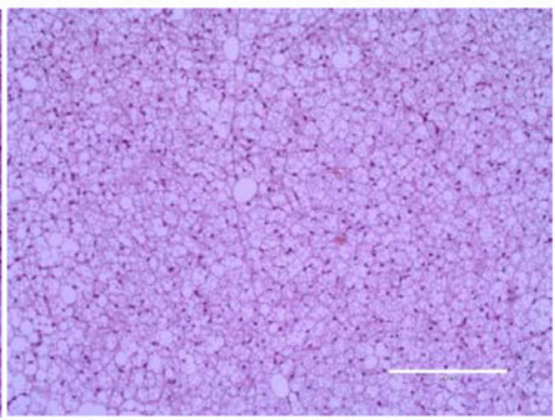

C
HFD+MA

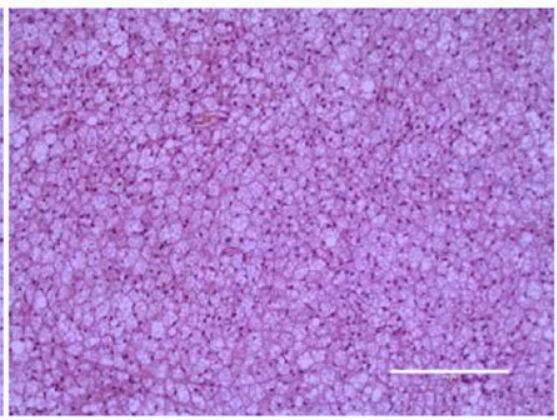

D
F

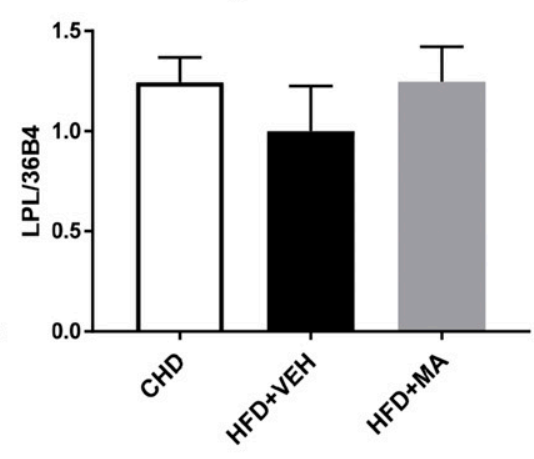

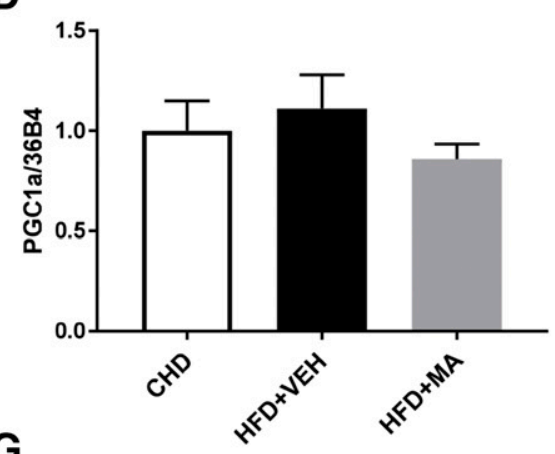

G

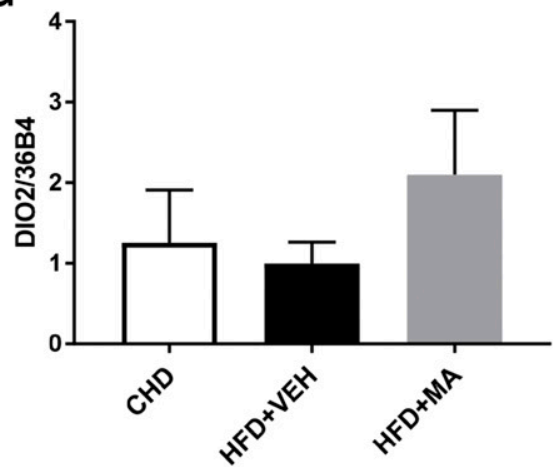

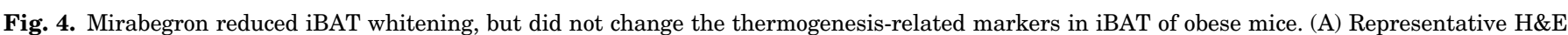

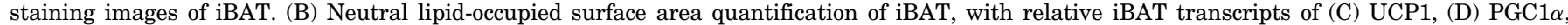

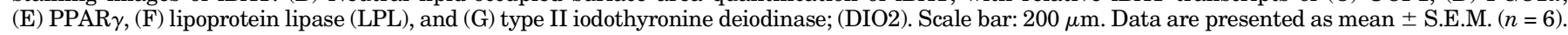

with the changes in iWAT morphology and UCP1 protein levels, iWAT UCP1 mRNA levels were also higher (13-fold) in the mice treated with HFD + MA (Fig. 5D). HFD + MA also increased the expression of CIDEA and carnitine palmitoyltransferase 1 (CPT1) compared with HFD + VEH (Fig. 5, E and $\mathrm{F}$ ). In addition, the leptin mRNA expression in iWAT was slightly reduced by the HFD + MA treatment (Fig. 5G), which concurs with reduced iWAT mass. No difference was found in mRNA expression of elongation of very long chain fatty acids 3 (ELOVL3), PRDM16, medium-chain acyl-CoA dehydrogenase (MCAD), adipose triglyceride lipase (ATGL), and hormone-sensitive lipase (HSL) (Fig. 5, H-L).

\section{Discussion}

Increasing energy expenditure through stimulation of $\beta 3-\mathrm{AR}$ in BAT might be an effective approach to treat obesity and metabolic syndrome (Ursino et al., 2009; Mund and Frishman, 2013). In the current study, we first investigated whether mirabegron could up-regulate brown fat activation-related markers in a mouse brown preadipocyte cell line and 3T3-L1 white preadipocytes. In mouse brown preadipocytes, we found that mirabegron significantly enhanced UCP1 gene expression at higher doses $(0.3$ and $3 \mu \mathrm{g} / \mathrm{ml})$, which was comparable to CL316243, a well-known $\beta 3$-AR agonist.

In 3T3-L1 white adipocytes, we observed that mirabegron up-regulated UCP1, PGC1 $\alpha$, and TMEM26, which are beige specific markers. $\beta 3$-AR is known to activate the protein kinase A/p38/mitogen-activated protein kinase signaling pathway, which consequently increases UCP1 promoter activity and further induces UCP1 gene expression (Kissig et al., 2016). Collectively, our in vitro study demonstrated that mirabegron increased the transcripts for brown fat and beige related markers in mouse brown preadipocyte and white preadipocyte cell lines, which might further enhance the thermogenic activity of these cells.

In the animal study, we found that the delivery of mirabegron to iBAT reduced body weight gain and fat mass in mice fed HFD. Importantly, the mirabegron treatment ameliorated 


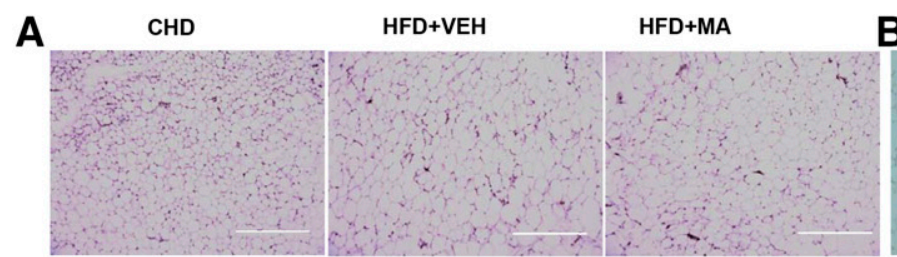

B $\quad$ CHD

HFD+VEH

HFD+MA
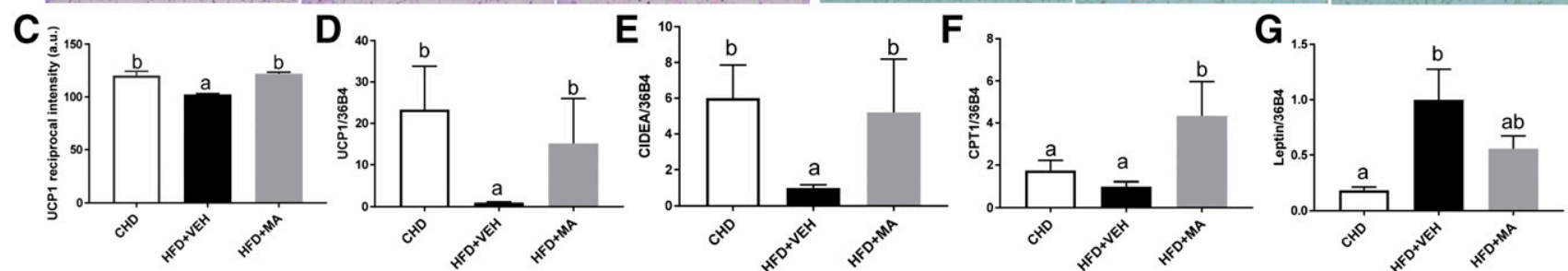

H

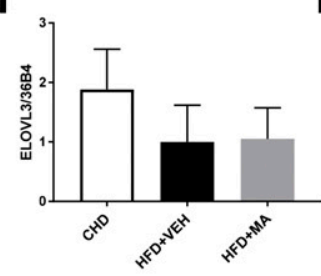

1

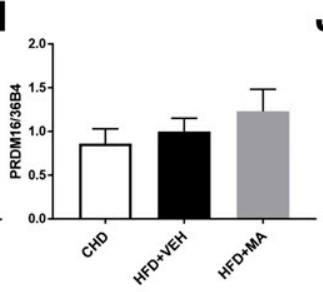

$\mathrm{J}$

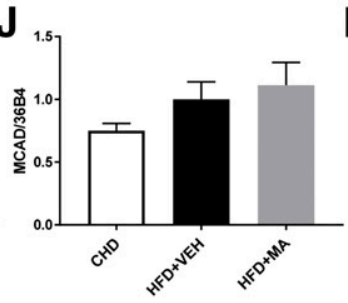

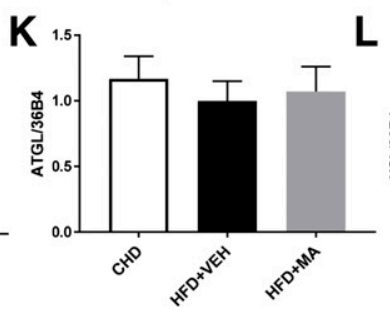

$\mathbf{L}$

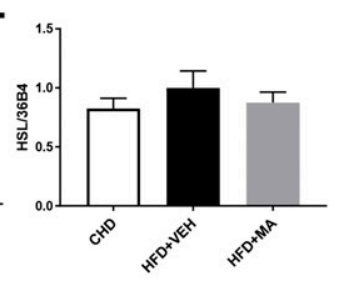

Fig. 5. Mirabegron-treated mice had increased transcription of thermogenesis-related markers and browning of iWAT. (A) Representative H\&E staining images of iWAT. Scale barL $400 \mu \mathrm{m}$. (B) Immunohistochemical analysis of UCP1 in iWAT. Scale bar: $200 \mu \mathrm{m}$. (C) Reciprocal intensity of UCP1 in iWAT, with relative iWAT mRNA levels of (D) UCP1, (E) CIDEA, (F) CPT1, (G) leptin, (H) elongation of very long chain fatty acids protein 3 (ELOVL3), (I) PRDM16, (J) medium-chain acyl-CoA dehydrogenase (MCAD), (K) adipose triglyceride lipase (ATGL), and (L) HSL. Data are presented as mean \pm S.E.M. $(n=6)$. Bars without a common superscript differ, $P<0.05$.

BAT "whitening” caused by HFD feeding and enhanced beige adipocyte formation in iWAT.

Obesity is associated with a dysfunction of BAT (Roberts-Toler et al., 2015). Specifically, HFD can lead to a BAT whitening phenotype that is characterized by mitochondrial dysfunction and large lipid droplet accumulation in brown adipocytes (Shimizu et al., 2014; Shimizu and Walsh, 2015). One recent study demonstrated that BAT whitening was attributed to the reduction of vascular endothelial growth factor a (VEGFa) signaling caused by HFD feeding (Shimizu et al., 2014). It would be interesting to investigate whether mirabegron regulates VEGFa signaling in a future study.

The iBAT UCP1 mRNA levels were similar among three groups, which is consistent with one study that reported that CL316243 improved BAT morphology but did not affect UCP1 gene expression (Xiao et al., 2015). In fact, one study revealed that even the strongest browning stimulus, cold, did not substantially increase UCP1 expression in iBAT when mice were housed at about $20^{\circ} \mathrm{C}$, because BAT is already fully differentiated and the density of UCP1 reaches the highest capacity in brown adipocytes (Kalinovich et al., 2017).

In addition to the effects on iBAT, mirabegron stimulated browning of iWAT as indicated by the histology and gene expression data, which are consistent with other studies showing that chronic $\beta 3-\mathrm{AR}$ agonist administration led to the presence of multilocular lipid droplets in WAT(HimmsHagen et al., 2000) and enhanced the expression of UCP1 in WAT depots (Merlin et al., 2016). Similar to other $\beta 3$-AR agonist-mediated transcriptional regulation, the binding of mirabegron to WAT $\beta 3$-AR may elevate intracellular cAMP levels, which trigger intracellular signaling cascades such as activation of protein kinase A and phosphorylation of p38 mitogen-activated protein kinase, resulting in activation of transcription machinery and recruitment of transcription factors for UCP1 and other thermogenic genes (Merlin et al., 2016).

There are very limited data showing the effect of mirabegron on adipose tissue and other metabolic active tissues. One study showed that administering mirabegron $(10 \mathrm{mg} / \mathrm{kg}$ per day) via gavage for 2 weeks decreased epididymal fat by $34 \%$ in obese C57BL/6 mice (Calmasini et al., 2017). Notably, a recent human study reported that mirabegron treatment ( $50 \mathrm{mg} /$ day for 10 weeks) induced UCP1, TMEM 26 , and CIDEA expression in the subcutaneous WAT of obese subjects although their body weights were not significantly changed (Finlin et al., 2018), which supports the findings of our studies.

Interestingly, we found that mirabegron significantly improved glucose tolerance and insulin sensitivity. It is unclear at this point how mirabegron acts to favorably impact glucose homeostasis and insulin signaling, but we speculate that stimulation of fat oxidation by $\beta 3$-AR agonists may quickly decease intracellular fatty acyl $\mathrm{CoA}$ and diacylglycerol, which are known to inhibit insulin signaling (Arch, 2002). Indeed, our results suggest that mirabegron may enhance fatty acid oxidation by increasing the expression of CPT1 gene (Fig. $5 \mathrm{~F}$ ), one of the key enzymes regulating beta oxidation of long chain fatty acids.

In addition, mounting evidence suggests that BAT functions as a glucose clearing organ (Festuccia et al., 2011; Lee et al., 2016). Therefore, the antidiabetic effects of mirabegron may be partially attributed to the release of brown adipokines by BAT, such as insulin-like growth factor I, C-X-C motif chemokine ligand-14, and fibroblast growth factor-21 (Townsend and Tseng, 2012; Villarroya et al., 2013; Cereijo et al., 2018). Furthermore, the reduction of adiposity per se may improve glucose tolerance and insulin sensitivity. Finally, similar to 
mirabegron, chronic administration of other $\beta 3$-AR agonists has been shown to improve glucose homeostasis as well (Kim et al., 2006; Fu et al., 2008). Further studies are warranted to investigate these mechanisms.

Our study has several limitations. First, calorimetry was not performed in the animal study, so we cannot directly verify that mirabegron increased energy expenditure in these obese mice. In addition, the cardiovascular effects of mirabegron were not tested in our in vivo models. As any $\beta 3$-AR agonist may have an off-target cardiovascular effect (Andersson, 2017), the safety of mirabegron on the cardiovascular system should be carefully examined. Notably, one study has found that mirabegron did not increase cardiovascular risk at a dose of $100 \mathrm{mg}$ in healthy subjects, but higher doses (150 and $200 \mathrm{mg}$ ) increased blood pressure (Loh et al., 2019). Finally, the mice were only placed in $22^{\circ} \mathrm{C}$, not in thermonueutrality. As housing temperature has marked effects on rodent physiology, it has been argued that metabolic studies in mice should be performed under thermoneutral conditions (Harms and Seale, 2013; Fischer et al., 2018).

In summary, the results of our current studies indicate that mirabegron can enhance UCP1 expression in mouse brown adipocytes and white adipocytes and promote browning of iWAT, which are associated with beneficial effects in protecting against HFD-induced obesity and improving glucose tolerance and insulin sensitivity. Our findings support further investigation of whether mirabegron could stimulate the recruitment of beige cells and improve glucose homeostasis in human subjects.

\section{Acknowledgments}

The authors thank Dr. Jie Liu (Texas Tech University) for her help in the animal study.

\section{Authorship Contributions}

Participated in research design: Hao, Wang.

Conducted experiments: Hao, Scott, Abbasi, Zu, Khan, Wu, Wang. Performed data analysis: Hao, Scott, Abbasi, Zu, Khan, Yang, Wu, Zhao, Wang.

Wrote or contributed to the writing of the manuscript: Hao, Scott, Abbasi, Khan, Zu, Yang, Wu, Zhao, Wang.

\section{References}

Andersson KE (2017) Pharmacology: cardiovascular effects of mirabegron. Nat Rev Urol 14:587-588.

Arch JR (2002) $\beta$ (3)-Adrenoceptor agonists: potential, pitfalls and progress. Eur $J$ Pharmacol 440:99-107.

Arch JR (2011) Challenges in $\beta$ (3)-adrenoceptor agonist drug development. Ther Adv Endocrinol Metab 2:59-64.

Arch JR (2015) Horizons in the pharmacotherapy of obesity. Curr Obes Rep 4:451-459.

Asano H, Kanamori Y, Higurashi S, Nara T, Kato K, Matsui T, and Funaba M (2014) Induction of beige-like adipocytes in 3T3-L1 cells. J Vet Med Sci 76:57-64.

Calmasini FB, de Oliveira MG, Alexandre EC, da Silva FH, da Silva CPV, Candido TZ, Antunes E, and Mónica FZ (2017) Long-term treatment with the beta-3 adrenoceptor agonist, mirabegron ameliorates detrusor overactivity and restores cyclic adenosine monophosphate (cAMP) levels in obese mice. Neurourol Urodyn 36:1511-1518.

Cannon B and Nedergaard J (2004) Brown adipose tissue: function and physiological significance. Physiol Rev 84:277-359.

Cawley J and Meyerhoefer C (2012) The medical care costs of obesity: an instrumental variables approach. $J$ Health Econ 31:219-230.

Cereijo R, Gavaldà-Navarro A, Cairó M, Quesada-López T, Villarroya J, Morón-Ros S, Sánchez-Infantes D, Peyrou M, Iglesias R, Mampel T, et al. (2018) CXCL14, a brown adipokine that mediates brown-fat-to-macrophage communication in thermogenic adaptation. Cell Metab 28:750-763.e6.

Cypess AM, Lehman S, Williams G, Tal I, Rodman D, Goldfine AB, Kuo FC, Palmer EL, Tseng YH, Doria A, et al. (2009) Identification and importance of brown adipose tissue in adult humans. N Engl J Med 360:1509-1517.

Cypess AM, Weiner LS, Roberts-Toler C, Franquet Elía E, Kessler SH, Kahn PA, English J, Chatman K, Trauger SA, Doria A, et al. (2015) Activation of human brown adipose tissue by a $\beta 3$-adrenergic receptor agonist. Cell Metab 21:33-38.
Dehvari N, da Silva Junior ED, Bengtsson T, and Hutchinson DS (2018) Mirabegron: potential off target effects and uses beyond the bladder. $\mathrm{Br} J$ Pharmacol 175:4072-4082.

Emorine LJ, Marullo S, Briend-Sutren MM, Patey G, Tate K, Delavier-Klutchko C, and Strosberg AD (1989) Molecular characterization of the human beta 3-adrenergic receptor. Science 245:1118-1121.

Festuccia WT, Blanchard PG, and Deshaies Y (2011) Control of brown adipose tissue glucose and lipid metabolism by PPAR $\gamma$. Front Endocrinol (Lausanne) 2:84

Finlin BS, Memetimin H, Confides AL, Kasza I, Zhu B, Vekaria HJ, Harfmann B, Jones KA, Johnson ZR, Westgate PM, et al. (2018) Human adipose beiging in response to cold and mirabegron. JCI Insight 3:e121510.

Fischer AW, Cannon B, and Nedergaard J (2018) Optimal housing temperatures for mice to mimic the thermal environment of humans: an experimental study. Mol Metab 7:161-170.

Flegal KM, Kruszon-Moran D, Carroll MD, Fryar CD, and Ogden CL (2016) Trends in obesity among adults in the United States, 2005 to 2014. JAMA 315.2284-2291.

Fu L, Isobe K, Zeng Q, Suzukawa K, Takekoshi K, and Kawakami Y (2008) The effects of beta(3)-adrenoceptor agonist CL-316,243 on adiponectin, adiponectin receptors and tumor necrosis factor-alpha expressions in adipose tissues of obese diabetic KKAy mice. Eur J Pharmacol 584:202-206.

Hao L, Kearns J, Scott S, Wu D, Kodani SD, Morisseau C, Hammock BD, Sun X, Zhao L, and Wang S (2018) Indomethacin enhances brown fat activity. J Pharmacol Exp Ther 365:467-475.

Harms M and Seale P (2013) Brown and beige fat: development, function and therapeutic potential. Nat Med 19:1252-1263.

Herschorn S, Chapple CR, Abrams P, Arlandis S, Mitcheson D, Lee KS, Ridder A, Stoelzel M, Paireddy A, van Maanen R, et al. (2017) Efficacy and safety of combinations of mirabegron and solifenacin compared with monotherapy and placebo in patients with overactive bladder (SYNERGY study). BJU Int 120:562-575.

Himms-Hagen J, Melnyk A, Zingaretti MC, Ceresi E, Barbatelli G, and Cinti S (2000) Multilocular fat cells in WAT of CL-316243-treated rats derive directly from white adipocytes. Am J Physiol Cell Physiol 279:C670-C681.

Kalinovich AV, de Jong JM, Cannon B, and Nedergaard J (2017) UCP1 in adipose tissues: two steps to full browning. Biochimie 134:127-137.

Kazak L, Chouchani ET, Jedrychowski MP, Erickson BK, Shinoda K, Cohen P, Vetrivelan R, Lu GZ, Laznik-Bogoslavski D, Hasenfuss SC, et al. (2015) A creatinedriven substrate cycle enhances energy expenditure and thermogenesis in beige fat. Cell 163:643-655

Kim DD and Basu A (2016) Estimating the medical care costs of obesity in the United States: systematic review, meta-analysis, and empirical analysis. Value Health 19:602-613.

Kim H, Pennisi PA, Gavrilova O, Pack S, Jou W, Setser-Portas J, East-Palmer J, Tang Y, Manganiello VC, and Leroith D (2006) Effect of adipocyte beta3-adrenergic receptor activation on the type 2 diabetic MKR mice. Am J Physiol Endocrinol Metab 290:E1227-E1236.

Kiso T, Namikawa T, Tokunaga T, Sawada K, Kakita T, Shogaki T, and Ohtsubo Y (1999) Anti-obesity and anti-diabetic activities of a new $\beta 3$ adrenergic receptor agonist, $\quad(S)-(Z)-[4-[[1-[2-[(2-h y d r o x y-3-p h e n o x y p r o p y l)]$ amino]ethyl]-1-propenyl] phenoxy] acetic acid ethanedioic acid (SWR-0342SA), in KK-Ay mice. Biol Pharm Bull 22:1073-1078.

Kissig M, Shapira SN, and Seale P (2016) SnapShot: brown and beige adipose thermogenesis. Cell 166:258-258.e1.

Klein J, Fasshauer M, Ito M, Lowell BB, Benito M, and Kahn CR (1999) $\beta(3)$ Adrenergic stimulation differentially inhibits insulin signaling and decreases insulin-induced glucose uptake in brown adipocytes. J Biol Chem 274:34795-34802.

Klein J, Fasshauer M, Klein HH, Benito M, and Kahn CR (2002) Novel adipocyte lines from brown fat: a model system for the study of differentiation, energy metabolism, and insulin action. BioEssays 24:382-388.

Lee P, Bova R, Schofield L, Bryant W, Dieckmann W, Slattery A, Govendir MA Emmett L, and Greenfield JR (2016) Brown adipose tissue exhibits a glucoseresponsive thermogenic biorhythm in humans. Cell Metab 23:602-609.

Loh RKC, Formosa MF, La Gerche A, Reutens AT, Kingwell BA, and Carey AL (2019) Acute metabolic and cardiovascular effects of mirabegron in healthy individuals. Diabetes Obes Metab 21:276-284.

Merlin J, Evans BA, Dehvari N, Sato M, Bengtsson T, and Hutchinson DS (2016) Could burning fat start with a brite spark? Pharmacological and nutritional ways to promote thermogenesis. Mol Nutr Food Res 60:18-42.

Mund RA and Frishman WH (2013) Brown adipose tissue thermogenesis: $\beta 3$ adrenoreceptors as a potential target for the treatment of obesity in humans. Cardiol Rev 21:265-269.

Nguyen DH, Zhou T, Shu J, and Mao JH (2013) Quantifying chromogen intensity in immunohistochemistry via reciprocal intensity. Cancer InCytes 2:e34.

Omran Z (2017) Obesity: current treatment and future horizons. Mini Rev Med Chem 17:51-61.

Ozaki K, Sano T, Tsuji N, Matsuura T, and Narama I (2011) Carnitine is necessary to maintain the phenotype and function of brown adipose tissue. Lab Invest 91:704-710.

Roberts-Toler C, O'Neill BT, and Cypess AM (2015) Diet-induced obesity causes insulin resistance in mouse brown adipose tissue. Obesity (Silver Spring) 23:1765-1770.

Sacco E, Bientinesi R, Tienforti D, Racioppi M, Gulino G, D'Agostino D, Vittori M, and Bassi P (2014) Discovery history and clinical development of mirabegron for the treatment of overactive bladder and urinary incontinence. Expert Opin Drug Discov 9:433-448.

Shimizu I, Aprahamian T, Kikuchi R, Shimizu A, Papanicolaou KN, MacLauchlan S, Maruyama S, and Walsh K (2014) Vascular rarefaction mediates whitening of brown fat in obesity. $J$ Clin Invest 124:2099-2112.

Shimizu I and Walsh K (2015) The whitening of brown fat and its implications for weight management in obesity. Curr Obes Rep 4:224-229.

Townsend $\mathrm{K}$ and Tseng YH (2012) Brown adipose tissue: recent insights into development, metabolic function and therapeutic potential. Adipocyte 1:13-24. 
Townsend KL and Tseng YH (2014) Brown fat fuel utilization and thermogenesis. Trends Endocrinol Metab 25:168-177.

Tubaro A, Batista JE, Nitti VW, Herschorn S, Chapple CR, Blauwet MB, Siddiqui E Huang M, and Oelke M (2017) Efficacy and safety of daily mirabegron $50 \mathrm{mg}$ in male patients with overactive bladder: a critical analysis of five phase III studies. Ther Adv Urol 9:137-154.

Umekawa T, Yoshida T, Sakane N, Saito M, Kumamoto K, and Kondo M (1997) Antiobesity and anti-diabetic effects of CL316,243, a highly specific beta 3-adrenoceptor agonist, in Otsuka Long-Evans Tokushima fatty rats: induction of uncoupling protein and activation of glucose transporter 4 in white fat. Eur J Endocrinol 136:429-437.

Ursino MG, Vasina V, Raschi E, Crema F, and De Ponti F (2009) The beta3adrenoceptor as a therapeutic target: current perspectives. Pharmacol Res 59:221-234.

van Marken Lichtenbelt WD, Vanhommerig JW, Smulders NM, Drossaerts JM, Kemerink GJ, Bouvy ND, Schrauwen P, and Teule GJ (2009) Cold-activated brown adipose tissue in healthy men. $N$ Engl $J$ Med 360:1500-1508.

Villarroya J, Cereijo R, and Villarroya F (2013) An endocrine role for brown adipose tissue? Am J Physiol Endocrinol Metab 305:E567-E572.

Virtanen KA, Lidell ME, Orava J, Heglind M, Westergren R, Niemi T, Taittonen M, Laine J, Savisto NJ, Enerbäck S, et al. (2009) Functional brown adipose tissue in healthy adults. $N$ Engl $J$ Med 360:1518-1525.
Warner A, Kjellstedt A, Carreras A, Böttcher G, Peng XR, Seale P, Oakes N, and Lindén $\mathrm{D}$ (2016) Activation of $\beta 3$-adrenoceptors increases in vivo free fatty acid uptake and utilization in brown but not white fat depots in high-fat-fed rats. Am $J$ Physiol Endocrinol Metab 311:E901-E910.

Williams CA, Shih MF, and Taberner PV (1999) Sustained improvement in glucose homeostasis in lean and obese mice following chronic administration of the beta 3 agonist SR 58611A. Br J Pharmacol 128:1586-1592.

Xiao C, Goldgof M, Gavrilova O, and Reitman ML (2015) Anti-obesity and metabolic efficacy of the $\beta 3$-adrenergic agonist, CL316243, in mice at thermoneutrality compared to $22^{\circ} \mathrm{C}$. Obesity (Silver Spring) 23:1450-1459.

Yamaguchi O, Kakizaki H, Homma Y, Igawa Y, Takeda M, Nishizawa O, Gotoh M, Yoshida M, Yokoyama O, Seki N, et al. (2015) Safety and efficacy of mirabegron as 'add-on' therapy in patients with overactive bladder treated with solifenacin: a post-marketing, open-label study in Japan (MILAI study). BJU Int 116:612-622.

Address correspondence to: Dr. Shu Wang, Department of Nutritional Sciences, Texas Tech University, Box 41270, Lubbock, TX 79409-1270. E-mail: shu.wang@ttu.edu; or Dr. Lei Hao, Department of Nutritional Sciences, Texas Tech University, Box 41270, Lubbock, TX 79409-1270. E-mail: lei.hao@ttu.edu 